\title{
Filigrane
}

Écoutes psychothérapiques

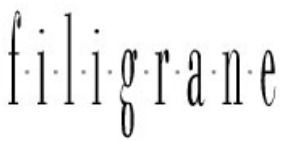

\section{Ce corps où la pensée s’est logée}

\section{Alain Lebel}

Volume 18, numéro 1, printemps 2009

Le corps. Sur le divan. Dans le fauteuil I

URI : https://id.erudit.org/iderudit/037717ar

DOI : https://doi.org/10.7202/037717ar

Aller au sommaire du numéro

\section{Éditeur(s)}

Revue Santé mentale au Québec

ISSN

1192-1412 (imprimé)

1911-4656 (numérique)

Découvrir la revue

\section{Citer cet article}

Lebel, A. (2009). Ce corps où la pensée s’est logée. Filigrane, 18(1), 7-27. https://doi.org/10.7202/037717ar

\section{Résumé de l'article}

Ce travail se veut une contribution aux réflexions sur ce passage du somatique au psychique. À partir de points de vue fournis par la théorie psychanalytique, par l'observation du bébé selon la méthode d'Esther Bick et par la pratique de la pédopsychiatrie, sera développée l'articulation entre les identifications primaires et secondaires qui signent l'influence déterminante des éprouvés corporels infantiles sur l'activité de représentation tout au cours de la vie. Nous partirons de textes de Freud tels l' "Esquisse... ", «Pulsions et destins des pulsions ", " Le Moi et le Ça ». Nous nous inspirerons aussi de nombreux théoriciens qui ont poursuivi dans le même sens, notamment Bion avec le concept de " rêverie maternelle " et Bick avec ceux de " peau psychique " et de " position adhésive » et leur interrelation avec les identifications primaires. Nous commenterons les positions d'auteurs contemporains tels Haag, Quinodoz, Lombardi, Ferrari et Balestrière qui réfléchissent à la place du corps et des affects dans le processus analytique même, de façon à mieux comprendre ce mouvement essentiel à la vie psychique qu'est celui qui part du pulsionnel en s'étayant sur le corps, de façon à alimenter l'activité psychique et la créativité humaine. Des exemples cliniques permettront de considérer les aléas de certains états primitifs du développement physique et psychique et ainsi de mieux saisir comment se mettent en place les articulations entre psyché et soma, les défauts qui peuvent en découler et faire l'objet d'un travail au cours du processus analytique.
Ce document est protégé par la loi sur le droit d'auteur. L’utilisation des services d’Érudit (y compris la reproduction) est assujettie à sa politique d'utilisation que vous pouvez consulter en ligne.

https://apropos.erudit.org/fr/usagers/politique-dutilisation/ 


\title{
Ce corps \\ où la pensée s'est logée
}

\author{
alain lebel
}

Ce travail se veut une contribution aux réflexions sur ce passage du somatique au psychique. À partir de points de vue fournis par la théorie psychanalytique, par l'observation du bébé selon la méthode d'Esther Bick et par la pratique de la pédopsychiatrie, sera développée l'articulation entre les identifications primaires et secondaires qui signent l'influence déterminante des éprouvés corporels infantiles sur l'activité de représentation tout au cours de la vie. Nous partirons de textes de Freud tels l' « Esquisse... », «Pulsions et destins des pulsions», «Le Moi et le Ça». Nous nous inspirerons aussi de nombreux théoriciens qui ont poursuivi dans le même sens, notamment Bion avec le concept de «rêverie maternelle» et Bick avec ceux de «peau psychique» et de «position adhésive» et leur interrelation avec les identifications primaires. Nous commenterons les positions d'auteurs contemporains tels Haag, Quinodoz, Lombardi, Ferrari et Balestrière qui réfléchissent à la place du corps et des affects dans le processus analytique même, de façon à mieux comprendre ce mouvement essentiel à la vie psychique qu'est celui qui part du pulsionnel en s'étayant sur le corps, de façon à alimenter l'activité psychique et la créativité humaine. Des exemples cliniques permettront de considérer les aléas de certains états primitifs du développement physique et psychique et ainsi de mieux saisir comment se mettent en place les articulations entre psyché et soma, les défauts qui peuvent en découler et faire l'objet d'un travail au cours du processus analytique.

«La pensée s'origine toujours dans un corps... et ajoutait Leclerc, dans une expérience, dans une sensibilité, dans une époque et évidemment dans un sexe. Tout ce que cela signifie, mais c'est immense, c'est que nous ne sommes pas Dieu. [...] Tout ce que l'on peut penser, dire, écrire et croire, on le doit au fait d'être en vie, c'est-à-dire, charnel, c'est-à-dire mortel. »

In Passions d'Annie Leclerc, Nancy Huston, Actes Sud/Leméac, 2007, p.110

«Mon hypothèse est que le corps est l'objet principal de l'esprit et sa première réalité. »

A.B. Ferrari, 2004, p. 37

\section{Introduction}

e corps précède la pensée. En effet, dès la vie fœtale, résultat de la rencontre intime des parents, un corps nous est accordé comme une donnée brute, roc ou assise avec lequel nous devrons composer durant toute notre existence. Une fois la naissance et le choc du commencement dépassés, chacun y greffera, de 
façon graduelle, un sentiment d'être soi, rivé et contenu dans l'enveloppe de son corps. Dès lors, l'amalgame corps-esprit se complexifiera au fil et au rythme du développement par une interaction continue de l'un et de l'autre.

Puisque la sensorialité prédomine chez le bébé naissant, la psyché croîtra en s'appuyant sur les fonctions corporelles (Anzieu, 1985). De fait, l'apparition de l'activité de représentation psychique soutient l'emmagasinage des expériences corporelles pour une éventuelle mise en sens, offrant en particulier une contenance face à la poussée vitale et aux mouvements pulsionnels parfois chaotiques et excessifs des sensations initiales. Source primordiale pour l'activité de mentalisation, l'axe corps-esprit ne pourra cependant s'épanouir et l'individu trouver sa trajectoire dans un univers relationnel aléatoire que s'il est aiguillé par une «figure maternelle» capable d'une rêverie qui sache prêter sens et relief à ses expériences.

De façon semblable, dans chaque entreprise psychothérapique, un jeu s'ouvre et s'engage entre deux sujets qui se côtoient, chacun avec son corps, ses pensées, ses sentiments, ses sensations, ses identités psychologique et sexuelle. Ogden $(1994,2001)$ invite à tenir compte de la tension dynamique existant dans le rapport analysant/analyste, suivant en ceci le modèle décrit par Winnicott et selon lequel un bébé seul n'existe pas. Cette position oblige, d'entrée de jeu, à reconsidérer les messages corporels tels qu'ils influent sur l'activité de pensée et s'entremêlent au code d'échange verbal. La construction des identités corporelle et psychique transite nécessairement par les identifications et les défenses primitives de l'analysant, et de l'analyste tout autant, lequel doit accepter de retourner fréquenter les siennes. Le travail analytique implique toutefois une activité psychique qui permettra un passage des processus primaires de la pensée vers une secondarisation de ceux-ci, de façon à aider à revitaliser, sinon créer chez l'analysant des voies de communication entre son corps et sa psyché, chemins souvent ignorés ou court-circuités au profit de l'intellectualisation. C'est dans ce contexte qu'une forme d'intersubjectivité s'installe, laquelle emprunte sans doute des couleurs et des aspects plus ou moins explicites d'une réédition n'apparaissant que dans l'après-coup.

Dans un livre regroupant ses écrits, Ferrari (2004) défend l'importance du corps dans le processus analytique théorisé sous l'angle d'«objet concret original»: le corps dans sa corporéité même s'offre comme objet primaire pour la psyché. Pour cet auteur, ce noyau physique original est ce qui fait la différence pour chaque être humain, plus encore, il intervient avant l'inscription de processus relationnels.

Ainsi, de prime abord, analyste et analysant sont confrontés à leurs différences, tant il est vrai qu'en clinique, le premier contact passe souvent par la seule présence de la voix. Celle-ci est unique et en dit beaucoup sur la personne, sur son âge et ses dispositions, par sa modulation chantante ou inexpressive, par son rythme pausé ou saccadé, son tonus dynamique ou affaissé. Au premier rendezvous, le corps entier s'impose par sa tenue, l'état de la personne se lit dans son regard, le parfum qu'elle dégage, l'harmonie de sa posture. L'analyste réagit à qui se présente à lui par des mouvements identificatoires ou contre-identificatoires très personnels, le plus souvent inconscients. La couleur des yeux d'une telle, la 
rudesse de la voix d'un autre, l'affirmation de la masculinité ou de la féminité, de la beauté, de l'étrangeté ou même d'une certaine laideur, l'aménagement de l'intégrité physique avec ses imperfections et ainsi de suite, constituent autant de données qui ne prendront tout leur sens qu'ultérieurement en évoquant des attributs physiques et psychiques propres à ses objets internes. Du côté de l'analysant, les remarques anodines ou détournées sur le décor, la luminosité de la pièce ou l'odeur du lieu, sur la qualité de la main tendue à l'accueil, serrée ou moite, sur la présence physique du thérapeute, son âge ou son style d'habillement, voire même sur la concordance des couleurs portées par chacun, témoignent d'une corporéité, d'une présence impliquant tous les sens. D'emblée, un rapport unique avec le dispositif analytique s'installe avec ce que ce cadre appelle d'intimité, et de là, de limites interpersonnelles à élaborer et de distances à pressentir et respecter.

L'analysant s'amène à l'intérieur de l'espace analytique avec ses a priori concernant l'analyste et le processus analytique, lesquels sont doublés par des attentes de soulagement qui concernent souvent le corps soumis à des douleurs physiques, de l'hypochondrie ou à l'emprise d'addictions. Un grand nombre de difficultés énoncées directement par l'analysant ou à travers une plainte font ainsi référence à son incapacité à jouir de la vie ou ressentir du plaisir. Cette capacité de jouissance, d'abord corporelle, se trouve attisée par diverses sources sensorielles, ce qui n'est pas sans créer un paradoxe puisque la douleur psychique sera temporairement reléguée au second plan. Ceci n'est pas surprenant quand on sait combien le physique et le psychique se confondent au début de la vie. Le fœtus expérimente les premières traces d'inscriptions sensorielles très tôt dans son développement, de l'odorat, du goût, du toucher, ou encore de l'ouïe, traces qui pourront prendre un sens dans un deuxième temps, dans l'après coup (Bizzozero, 2007).

Mes formations en psychanalyse, en pédopsychiatrie et à l'observation des bébés selon la méthode de Bick, par le croisement des pensées qui s'est opéré, m'ont ouvert à ce questionnement. Ces observations centrées sur le bébé aiguisent les capacités d'attention des psychothérapeutes avec les personnes qui se présentent en consultation et sensibilisent au fait que le corps constitue un puissant moyen de communication par le biais de l'attention portée aux manifestations de tension interne. Au cours de ce travail qui se veut une contribution aux réflexions sur ce passage crucial du somatique au psychique, c'est à partir de ces différents points de vue que j'examinerai l'articulation entre les identifications primaires et secondaires qui prennent en compte l'influence qu'exerceront ces aspects corporels infantiles tout au cours de la vie.

Nous retournerons aux textes de Freud qui, dès «L'Esquisse... » en 1895, a cherché à saisir les mécanismes en cause entre le corps et l'esprit, recherche qu'il a menée jusqu'à la fin de son œuvre, jalonnée par des textes comme «Pulsions et destins des pulsions » (1915), «Le Moi et le Ça » (1923). À sa suite, de nombreux théoriciens ont poursuivi dans ce sens à sa suite, notamment Bion avec le concept 
de rêverie maternelle (1962) et Bick (1968) avec ceux de peau psychique et de position adhésive et leurs interrelations avec les identifications primaires. Plus récemment, des auteurs contemporains tels Quinodoz (2002), Lombardi (2008), Ferrari (2004) et Balestrière (2008) ont repris cet examen de la place du corps et des affects dans le processus analytique même, de façon à mieux comprendre ce mouvement essentiel à la vie psychique qu'est celui qui part du pulsionnel et, s'étayant sur les éprouvés corporels, alimente l'activité mentale de représentation et la créativité humaine.

L'expérience de l'observation des nourrissons — dont j'ai rendu compte ici et ailleurs (Lebel, 2000, 2008) —, est d'autant plus riche d'enseignement qu'elle offre le privilège unique de nous mettre en contact avec le corps du bébé qui découvre en quelque sorte l'espace de son corps, son corps dans l'espace alors que celui-ci est confronté à des sensations physiques brutes qui attendent que les qualités psychiques de l'esprit l'aident à endiguer ce flot sensoriel. Graduellement, les réflexions portées sur les débuts de la vie ont permis l'émergence d'une double source à la base du développement de la personnalité : le monde relationnel et l'expérience de la solitude. Le bébé soumis à un rythme de présence et d'absence maternelle et paternelle sera amené à développer des capacités psychiques différentes liées à cette alternance. En effet, force est de constater qu'il s'installe de façon précoce pour le bébé, lorsqu'il est seul, un rapport à son propre corps, indépendant du mode relationnel.

Plus tard, à l'adolescence, durant une grossesse ou au cours d'une maladie, ce rapport au corps s'intensifie ou même s'exacerbe: l'appareil à penser aura à affronter les changements physiques imprévisibles qui découlent de ces états et il en résulte souvent une confusion importante dans le monde intérieur, confusion perdurant même durant la vie adulte. Le rassemblement des parties de la personnalité, suivant un processus intégratif plus ou moins laborieux, génèrera un sentiment d'être une personne «totale» avec une histoire personnelle qui fait sens et qui procure une impression de continuité et d'authenticité de son être corporel. En découvrant qu'il $a$ un corps mais en réalisant qu'il est aussi ce corps, l'analysant est ainsi à même de réappréhender son «self», avec le sentiment d'être un «sujet» face à lui-même, habitant un espace suffisant pour l'action et la créativité tout en reconnaissant l'autre en toute sécurité.

\section{Position et identité adhésives}

Une des propositions de cet article est de considérer les états primitifs du développement physique et psychique pour ainsi tenter de saisir les articulations entre la psyché et le somatique, les défauts qui peuvent en découler et rester par la suite difficilement accessibles durant le travail analytique. Nous sommes condamnés, réalité oblige, à avoir recours à des métaphores pour approcher des états dits originaires et les revoir à la lumière des théories existantes.

À partir de 1948, Esther Bick, psychanalyste anglaise, a développé la méthode d'observation des bébés dont la conceptualisation est largement redevable à la 
psychanalyse. L'apport théorique qu'elle nous a laissé postule qu'au début de la vie, une première forme d'identification par adhésivité intervient — il s'agit d'une identité de surface — , qui amène le bébé à développer une peau psychique. Cette identification qui nécessite une mise en sens active des sensations corporelles du bébé est alimentée par un «objet» maternel suffisamment bienveillant et contenant dont le résultat sera une intériorisation progressive du lien à une figure maternelle en autant qu'elle soit assez disponible physiquement et psychiquement.

Bick (1968) situe la position adhésive «normale» avant toute forme d'introjection, la rapportant à un moment antérieur aux positions schizo-paranoïde et dépressive décrites par Melanie Klein. Durant cette période, elle postule que l'angoisse est vécue sur un mode catastrophique, le bébé vivant encore dans un état de non-intégration, comparé aux moments de désorganisation ou de désintégration retrouvés plus tard avec les angoisses de perte typiques de la position dépressive ou de persécution propres à la position schizo-paranoïde. Ciccone (2001) a récemment élargi l'aspect défensif de l'adhésivité, défense à laquelle peut avoir recours la psyché à tout moment de son évolution pour éviter la menace inhérente à des expériences de séparation, d'individuation et lors de surcharges émotionnelles vécues en franchissant des étapes de transition de la vie. Ainsi, l'adhésivité ne traite pas la terreur; elle colmate tout au plus un moment la brèche ouverte par un trop plein d'angoisse ou un choc inassimilable.

Nous savons maintenant qu'il existe une continuité dans les éprouvés physiologiques anté et post-nataux. In utero, le fœtus est soumis à un flot sensoriel précoce d'intensité variable: il sent, il goûte, il réagit aux sensations d'ordre tactile, thermique, proprioceptif et même visuel. Un premier espace intérieur — contenant physique - existe puisqu'il absorbe et rejette le liquide amniotique avec un rythme personnel (Bizzozero, 2007). Les expériences pré-natales gravent ainsi les toutes premières impressions, creusant un premier sillon non encore mentalisé. Il n'est donc pas étonnant qu'au tout premier stade de la vie du nourrisson, les ressentis corporels forment le point de cristallisation autour duquel va s'édifier notre sentiment d'identité.

Le bébé naissant doit apprendre à respirer et son organisme à supporter l'effet des forces gravitationnelles dont il était exempté jusqu'à sa naissance. Par la suite, l'image du corps prend forme à partir des sensations associées aux postures et aux mouvements des membres et de celles rencontrées par la peau et ses orifices, en particulier la bouche et les sphincters. Le portage par des personnes fiables apporte aux bébés une détente qui les met en état de capter et pouvoir absorber les informations du monde externe en toute sécurité. En les observant, nous constatons combien les registres affectif, cognitif et de communication sont sollicités et combien leur efficience varie en fonction de la qualité de la tenue et de la manipulation du bébé. S'ils prennent de l'expansion après des soins satisfaisants, l'activité dans chacun de ces registres s'amoindrit quand le bébé n'est plus tenu; les vocalises diminuent, le corps se tend ou au contraire s'affaisse et l'intérêt pour le monde environnant se réduit sans un support proximal attentif aux besoins de 
l'enfant. De telles fluctuations permettent aussi d'apprécier cette tension plus ou moins supportable associée aux premières expériences de solitude comparée au plaisir chez l'enfant à s'appuyer sur un objet maternel vivant et réceptif. Pour que ces phénomènes adviennent à travers un corps qui fait exister et informe un sujet, il faut que le jeune enfant dispose d'un objet fiable et attentif à filtrer les sources d'excitation qui risquent autrement de l'envahir. L'exemple de Diane, née et laissée en pouponnière à la naissance par sa mère, servira à illustrer ces propositions théoriques.

\section{Extrait de la première observation : Diane a 15 jours de vie, avec sa mère substitut}

C'est l'heure du bain. Annabelle achève de déshabiller Diane; ce n'est que lorsqu'elle est toute nue que Diane se met à pleurer fort. Annabelle met sa grande main sur le ventre du bébé en lui disant: "N'aie pas peur, je te tiens, tu ne vas pas tomber». Elle se calme et pleure à nouveau dès qu'Annabelle ne la touche plus et ainsi de suite. Le mouvement pour passer du comptoir à la baignoire déclenche des pleurs qui cessent dès qu'elle est immergée dans l'eau.

\section{Extrait de la deuxième observation : Diane a trois semaines. Un autre bain}

Annabelle soulève doucement Diane qui est étendue sur un tapis de sol, en prenant soin de bien soutenir le cou et la tête de l'enfant. Diane se recroqueville sur la poitrine d'Annabelle, poings serrés, sans pouvoir parvenir à s'agripper activement à celle-ci. Annabelle la dépose sur la serviette disposée sur le comptoir et aussitôt Diane vomit abondamment par vagues successives. Annabelle la redresse aussitôt, la regarde attentivement et, toujours inquiète, lui demande si c'est terminé. Je partage cette inquiétude en silence. Quand Annabelle quitte Diane des yeux tout juste le temps de me dire qu'elle a aussi de la diarrhée, elle vomit à nouveau, le corps secoué de spasmes, le visage grimaçant de dégoût. Annabelle s'occupe à nettoyer et changer la petite avec douceur et tendresse en l'emmaillotant. Diane regarde autour d'elle; j'observe ses yeux cernés et son air épuisé par l'effort fourni.

Se sentir tenu est une sensation corporelle de base essentielle, relayée par la qualité du toucher, du regard ou de l'enveloppement global procuré à l'enfant. Dans cet extrait, Diane, même blottie sur l'adulte, demeure crispée. Elle vomit lors d'un moment de transition et son corps tout entier traduit le chaos et cherche à communiquer son désarroi momentané à son entourage. Lorsque l'enfant est déposée nue sur la table, c'est l'absence soudaine de contact peau à peau qui semble déclencher ses pleurs. Diane est pourtant bien entourée par Annabelle qui pressent de façon intuitive la peur de tomber chez le bébé.

De telles observations avec en alternance des moments de tenue et de lâchage ont été faites dans de multiples dyades mère-bébé et Haag (1984) rapporte la présence de propos rassurants chez des mères capables de s'identifier à ce que peut 
vivre l'enfant. Diane nous laisse avec l'impression d'être affolée par cette sensation d'être vidée qui évoque alors à l'intérieur de l'observateur ce moment précis où, frappé d'une gastro-entérite, un sentiment de catastrophe nous envahit avec l'impression de se répandre et de craindre pour sa survie même.

Quand le bébé affronte un vécu d'une telle intensité — que certains qualifient d'angoisse agonistique — sans trouver de réponse dans son environnement ou sans pouvoir profiter de l'appui qui lui est offert, il aura recours à des défenses impliquant le corps. Parmi celles-ci, on notera tantôt l'immobilité, la prostration, yeux fermés, en serrant les poings comme pour repousser la menace, tantôt l'agrippement à une modalité sensorielle pour se tenir, que ce soit par le regard rivé à un stimulus visuel (lumière, chatoiement, brillance) ou par l'audition en s'attardant à un bruit, telle une musique, le chuintement de l'eau qui s'écoule ou le bruit des pas autour de lui, qui permet à l'enfant de fuir en quelque sorte la source de danger.

Le bébé est confronté à la tâche de réguler la pulsion selon le modèle économique de Freud - cette «poussée constante» (Freud, 1915) qui l'habite et l'assaille dès le début de la vie — avec les faibles moyens psychiques dont il dispose pour y parvenir. La position adhésive traduit cette tentative essentiellement physique de l'enfant pour s'agripper à une surface pour ne pas tomber, en quelque sorte d'y adhérer à défaut de pouvoir autrement la saisir. Toute menace potentielle suscite le besoin de rester collé — rappel instinctif de l'état d'être porté et contenu dans la matrice — en attendant qu'une «peau protectrice», psychique cette fois, se forme et joue son rôle de contenant régulateur, tant physiologique que psychique.

\section{Identification et narcissisme primaires}

Meltzer (1984) a proposé que le concept d'adhésivité de Bick pourrait être envisagé comme un mécanisme d'identification narcissique primitif qu'il a appelé identification adhésive. Freud (1917), dans Deuil et Mélancolie, avait pour la première fois parlé de l'identification narcissique qu'il considérait comme étant la plus originaire. Dans l'Abrégé (1938), il qualifie de «narcissisme primaire absolu» ce temps où la libido narcissique n'a pas encore commencé à se transformer en libido d'objet. Haag (1984, 1997), citant Tustin (1981) accepte l'idée «qu'avant le narcissisme primaire, il y a un état dominé par les sensations, centré sur le corps, qui forme la base du développement du self ».

Balestrière (2008), dans son questionnement sur les origines, reconnaît une place particulière au maternel dans la cure analytique en établissant un parallèle entre celle-ci et le cours de la vie d'un bébé. En effet, le rythme et l'espace des séances opèrent de façon à conférer à une série de moments d'intégration et de non-intégration le statut de «points d'agrippement» alors que chez le bébé, c'est l'alternance entre la présence relationnelle et la solitude qui contribuera à créer cet effet. Se rapportant au vécu des bébés, Athanassiou $(1982,1997)$ propose quant à elle une image de leur vie s'étalant en pointillés. Elle développe l'idée qu'ainsi, des points, plus ou moins espacés, résultant de stimulations sensorielles diverses, 
constituent une trajectoire où l'identité se trouve rassemblée et concentrée en autant de moments précis. Entre ces points, c'est l'expérience du vide, sans vie mentale, sans reconnaissance d'identité. Par la suite, avant l'acquisition d'une activité autoérotique mieux définie, comme celle de sucer son pouce, le bébé va utiliser les qualités de surface de l'objet en l'investissant pour constituer un premier niveau d'identification. Par exemple, le bébé «jouera» à coller sa main à plat sur sa bouche ou son visage, laissant l'impression de recréer pour lui-même un contact physique de peau à peau, tel qu'il vient de l'éprouver avec sa mère. Bick a saisi que cela ne se produisait pas à l'intérieur du bébé mais à la surface de son corps, une étape que Tustin a qualifié d'auto-sensuelle.

Un peu plus tard, les bébés observés attentivement montrent qu'ils reproduisent pour eux-mêmes les qualités du geste autour d'un soin, tel que l'objet maternel vient de le lui prodiguer de façon encore plus fine, témoignant du processus d'intériorisation en cours. Cette forme d'identification très primaire passe par le corps avant tout. Haag (2004) rapporte le jeu de mains d'un bébé avec le sein de sa mère, jeu repris quelques minutes plus tard, cette fois en traitant le doigt de sa mère serré dans sa main comme il le faisait avec son sein, et par la suite en serrant son propre doigt avec son autre main de manière similaire, reprenant et reproduisant à sa façon les soins prodigués par sa mère. C'est ainsi que des parties du corps du sujet s'identifient à l'objet. Ces identifications précoces, maintenant intériorisées, fonderont le moi-corps que chacun portera sa vie durant.

Ce passage de l'adhésif à l'introjection varie selon la qualité de la relation à l'objet, qualité fluctuante dans toute dyade et qui demande du temps pour s'ajuster. Le caractère adhésif persisterait dans des types de relations où les expériences de lâchage prédominent. L'adhésivité prendra une forme introjective permettant le développement de l'autoérotisme, à condition que la contenance maternelle assure une circulation des mouvements pulsionnels, émotionnels et conversationnels en sachant en régler la charge sans avoir recours à des ruptures brutales. Il se créera alors chez l'enfant le sentiment d'un «Soi en continuité » en même temps que s'ébaucheront les contours l'altérité. C'est l'essence du concept de «peau psychique».

Cette période du développement précoce en est une de grande vulnérabilité alors que ces mouvements incessants d'exploration peuvent mener aussi bien du côté de la créativité que de la désorganisation. Comme le dit Haag (1991), «le moment adhésif des identifications est à la fois sans pensée et aussi le moment du contact le plus intime, moment de la découverte, de l'invention, de l'illumination». L'art psychothérapeutique tient en ceci d'amener ce monde sensuel, corporel, dans la sphère du langage en veillant à opérer cette transformation de façon à perdre le moins possible la richesse de ces matériaux d'origine.

\section{Identification adhésive pathologique : défaillances du premier contenant}

Les failles dans l' «objet contenant optimal» nous conduisent à réfléchir aux défauts de constitution de cette peau psychique, défauts entraînant les phénomènes de «seconde peau » (Bick, 1968, 1986; Haag, 2002). 
Mitrani (2008) résume de façon éclairante la distinction à établir entre peau psychique et seconde peau: «La peau psychique est la structure intrapsychique résultant du développement à travers une expérience consistante avec l'objet maternel et l'introjection subséquente d'un objet contenant tandis que la seconde peau est une structure autosensuelle compensatoire qui sert à colmater la conscience intenable du sentiment de séparation d'avec l'objet et ayant pour effet d'étouffer le développement mental et émotionnel. Les phénomènes rapportables à une seconde peau se manifestent autant par des conduites autosensuelles que par des actions ou des équations symboliques concrètes qui prennent souvent racine ou sont calquées sur des manifestations physiques de l'environnement maternel ». Par extension, ils peuvent prendre la forme d'un mouvement perpétuel, d'une hyperactivité motrice ou verbale, d'un surinvestissement de la sphère intellectuelle ou sensorielle tels qu'on en retrouve dans les conduites toxicomaniaques, dans la masturbation précoce et compulsive ou l'auto-stimulation chez certains carencés ou certains autistes.

«À défaut d'une identification aux attributs mentaux de l'objet, la phénoménologie de l'identification adhésive pathologique se traduit par divers phénomènes persistants: la dépendance «en se collant à», les réactions d'effondrement à la séparation et à l'imitation étroite de l'apparence et du comportement de surface de l'objet» (Meltzer, 1975). Ces attitudes laissent entrevoir que les figures parentales sont d'une absolue nécessité pour l'enfant et dans ces cas l'enjeu est davantage celui de la disparition de l'objet plutôt que sa perte. L'effondrement de l'enfant lors de séparations mal préparées et assumées renvoie l'impression d'un arrachement sans recours possible à des défenses d'omnipotence plus évoluées, les modes de résolution de l'angoisse ne constituant pas encore une possibilité de réaction organisée. Un mimétisme s'installe à la place de l'effort nécessaire pour comprendre les états mentaux de l'autre; la tête du sujet est vide, il est incapable de penser, laissant même parfois croire à une intelligence moindre que son potentiel réel.

Au plan thérapeutique, les tentatives pour interpréter les projections tombent à plat et il faut plutôt chercher à élaborer les peurs primitives de chute dans le vide, de désintégration dans un espace infini, en offrant des points de repère très concrets concernant le corps, le temps et l'ancrage spatial.

Voici l'exemple de Catherine:

Catherine est arrivée à 18 mois dans cette bonne famille d'accueil après avoir vécu pendant six mois avec une mère déficiente intellectuelle et avoir ensuite "épuisé» deux familles d'accueil inexpérimentées. Catherine a été soumise à de graves incohérences dans les soins durant cette période initiale comme en témoignent les observations des services sociaux impliqués auprès de la famille. À son arrivée en consultation, on prenait pour acquis que Catherine souffrirait d'une déficience intellectuelle marquée, vu l'importance de ses retards. La mère d'accueil, avertie des difficultés persistantes de sommeil de l'enfant, en était rapidement venue à lui proposer un contact peau à peau, collant le bébé contre sa poitrine pour l'endormir, décision intuitive couronnée de succès. 
Si ce besoin de contact intime a cédé après plusieurs semaines, Catherine est demeurée d'une dépendance excessive vis-à-vis de sa mère d'accueil au quotidien. En vieillissant, Catherine a fait un usage au premier abord amusant de l'imitation des rires, des postures et des mimiques de sa mère d'accueil, mais la persistance de cette reproduction caricaturale, avec peu de réciprocité, laissa d'autant plus perplexe que le développement de l'enfant demeurait ralenti. Sa capacité à jouer seule était restreinte et elle se contentait le plus souvent de «manipuler» les jouets sans inventer ni s'engager dans aucun jeu.

En plus des aspects constitutionnels qui lui sont propres, le début de la vie de Catherine avec sa mère biologique, mère peu affectueuse et très négligente avec son bébé, a laissé des traces qui continuent d'entraver chez elle la constitution d'une peau psychique. Catherine n'a pas pu intérioriser un objet contenant, elle ne prenait que les aspects de surface de l'objet disponible sans arriver à leur créer une place à l'intérieur d'elle-même. Des enfants éparpillés qui «papillonnent» comme Catherine sont attirés par les stimuli les plus saillants sans organiser leurs perceptions de façon cohérente et ils demeurent dans la confusion. L'adhésivité et la recherche effrénée d'un objet auquel s'accrocher demeurent marquées chez Catherine. Son mimétisme cache mal la pauvreté de ses capacités de représentation. Ce manque se traduit autant dans la maladresse extrême de son corps, son incapacité à dessiner ou plus tard à écrire, de même qu'au plan psychique où, malgré la grande sensibilité de sa mère d'accueil et sa disponibilité à l'autre, Catherine n'arrive pas à trouver une profondeur qui lui ouvrirait l'accès à la symbolisation.

À ce sujet, Bégoin (1989) amène l'idée que l'assimilation symbolique de l'expérience relationnelle précoce contribue à la construction de l'identité. Toutefois, lorsque l'identité de base n'est pas assurée, le développement de la symbolisation se trouve entravé, d'où l'importance des premières identifications et la place faite à l'intersubjectivité matérialisée dans l'interaction mère-enfant qui soutient et promeut le développement de l'activité psychique. Winnicott (1971) a d'ailleurs soulevé cette hypothèse en proposant de mettre ensemble l'intégration du lien psyché-soma, le self et la fonction contenante.

\section{Du corps concret au corps habité}

Pour Freud (1915), la source de la pulsion se situe dans un processus somatique à partir d'un organe ou d'une partie du corps et l'excitation résultante sera représentée dans la vie psychique via cette pulsion. La fabrication de l'être psychique, qui est au fondement du sujet, suppose un investissement du Moi qui précède le choix d'objet, les craintes rattachées à la perte de l'objet ou à la castration. Je rejoins ici Balestrière (2008, p. 260) et Ferrari (2004) qui situent la force pulsionnelle dans un espace d'action potentielle d'où émaneront les perceptions, les sensations, les émotions et les pensées.

S. Klein (1980) avance, qu'à défaut d'un bon contenant extérieur stable, c'est le corps qui peut être utilisé comme objet de projection, avec le risque de conduire à la production de symptômes psychosomatiques. Lombardi (2008) propose alors 
d'approcher le corps dans la session analytique comme un moyen de travailler sur les défauts de la pensée. Cela passe par la nécessité de limiter les interprétations transférentielles pour une période souvent prolongée pour plutôt s'intéresser au transfert sur le corps propre, avec pour l'analysant, l'obligation de faire ou refaire, selon le cas, connaissance avec son corps.

Denis vient de fêter ses 16 ans. Cet adolescent autistique mais verbal est aux prises avec des angoisses envahissantes, tantôt de persécution, tantôt de confusion et d'incompréhension par rapport au monde dans lequel il vit. Lors de la séance rapportée ici, Denis traverse une période de crise où il voudrait mourir plutôt que de supporter plus longtemps «s a folie».

Ce jour-là, il apparaît dans un état d'esprit différent, se permettant de me regarder plus directement, ce qui est rare avec lui. Je ressens un malaise en apercevant un geste répétitif chez lui qui consiste à "pincer/rouler» la peau de ses maigres bras pendant qu'il me parle de sa crainte intense de vivre du rejet en lien avec l'idée de contacter à nouveau un "ami». Denis, qui aime beaucoup les humoristes, se met soudainement à rire en pensant à une phrase d'un de ceux-ci: «ma prof de maths est une cochonne». Je connais Denis depuis quatre ans et j'ai souvent été témoin de sa manière de prendre les mots dans leur sens le plus concret, ce qui suscite chez lui d'importants épisodes d'excitation et de confusion. Sa mimique angoissée m'incite alors à lui demander si quelque chose le préoccupe par rapport à son corps, en me reportant au fait qu'il a souvent été question entre nous d'angoisses concernant son intégrité corporelle.

Il s'engage alors un dialogue et une réflexion complexes sur sa propre constitution physique: il croit que sa peau recouvre et protège ses os qui, eux, contiennent les muscles. Il se questionne sur la dureté et la solidité des os, la texture du muscle et la présence du sang contenu dans les os, plutôt que dans les muscles, avec le risque de perdre son sang s'il se casse un os. Il demeure dans le même registre angoissé en parlant d'un cochon à la peau sale qu'on peut manger, mais qu'il serait incapable de tuer. Il se demande s'il est le seul à penser ainsi. Je réagis intérieurement à cette conception archä̈que et angoissante à propos de l'écoulement d'une substance corporelle vitale et je pense pour ma part à mes craintes et mon dégoût d'avoir à vider un animal de son sang, tout en me disant qu'en situation d'urgence ou de survie, j'y arriverais. Je lui dis même qu'il existe un métier, celui de boucher, qui aide ceux qui ont peur de tuer des animaux.

Même si je pouvais entrevoir le sens possiblement sexualisé attribué au terme de «cochonne» et que je lui ai tendu une perche à ce niveau en disant qu'il avait peut-être déjà entendu des amis ou des adultes faire référence à des choses sexuelles en parlant de «cochon ou de cochonneries» ou encore en référant aux aspects oraux et cannibaliques associés à l'idée de manger son professeur, je me doutais que Denis ne pouvait pas l'entendre ainsi ce jour-là. L'excitation visible chez lui témoignait plutôt d'un affaissement des différents niveaux de sens qui se trouvaient agglutinés et lourdement confondus. Il nous fallait repartir de la réalité purement concrète du corps. 
J'ai alors plutôt mis l'accent sur sa conception d'une peau qui ne protège pas l'intérieur du corps. Ce qu'il recherchait, c'était la dureté, la rigidité de l'os et non la souplesse de la peau. Cette rigidité de l'os signe en même temps sa fragilité puisque le risque de cassure et l'écoulement de la substance contenue dans l'os est à craindre, faisant surgir l'angoisse primitive sous-jacente qui consiste à «se vider de son sang », comme un animal tué, s'il se blesse. Son sentiment d'intégrité physique et psychique se trouvait condensé chez lui dans cette même représentation. Pour ce qui est d'un travail au niveau d'angoisses de type paranoïde-schizoïde, telles que dévorer ou tuer des animaux, ou encore d'un travail s'adressant à des angoisses de niveau plus œdipien ou sexualisé, comme en témoignait son désir de toujours vivre chez ses parents ou son intérêt pour les danseuses nues, il m'apparaissait que ces constructions ne lui servaient à rien à ce moment de crise. Elles ont par contre été reprises lors de rencontres subséquentes.

Winnicott $(1945,1949,1952,1971)$ nous rappelle que la perte de contact même momentanée entre la psyché et le soma engendre un sentiment de dépersonnalisation dont il a décrit les différents états en termes d'intégration, de désintégration et de non-intégration. C'est dans ce dernier état qu'il souligne que les parties de la personnalité non encore attachées ou rassemblées peuvent ainsi circuler et s'investir dans une relation inconnue avec l'environnement et permettre au sujet d'accéder, le moment venu, à un développement ultérieur.

Dans cette conversation psychothérapeutique avec Denis, le thérapeute a pris le parti de jouer ce rôle, soit de réfléchir tout haut à des aspects primitifs concernant les fonctions du corps de façon à permettre à cet adolescent d'éprouver et de rassembler ses sensations corporelles, pour que ce qui était «hors du soi», sans inscription psychique, puisse s'intégrer. Pour Bion (1957,1962), ce sont de telles parties psychotiques de la personnalité qui, si elles ne sont pas traitées, vont demeurer dans le registre asymbolique. La partie psychotique qui s'exprimera par la fragmentation et la projection de l'appareil sensoriel mène à une dissociation des éprouvés sensoriels qui ne peuvent plus fonctionner ensemble. Ces fixations puissantes sur le corps deviennent persécutrices et ont un caractère quasi autistiques du fait d'être fortement égocentrées.

Dans de telles situations, l'analyste doit faire appel à cette capacité constante à recourir intérieurement à ses ressentis contre-transférentiels corporels avec la visée de faciliter cette rencontre corps-esprit problématique pour l'analysant. Ceci vaut notamment lorsqu'il s'agit de faire saisir les différences entre les aspects sensoriel, sensuel et sexuel du monde, lesquels peuvent être facilement confondus chez certains patients. En plus d'avoir en lui-même de solides repères d'arrièreplan procurés par sa propre analyse, l'analyste doit aussi être alimenté par ses groupes de travail et son propre travail de réflexion et de recherche faisant appel à l'auto-analyse en rapport avec ses propres parties psychotiques, comme le souligne S. Klein (1980).

Hinshelwood, dans la présentation du livre de Ferrari (2004), fait le rapprochement entre le corps comme objet concret original et le concept d'identité 
adhésive de Bick. Il suit Ferrari qui a proposé l'idée importante de faire du corps concret un objet primaire pour la psyché, « un objet non symbolique qui développe sous certaines conditions, la capacité de penser et de symboliser», présent avant toute forme d'introjection. Du même souffle, Ferrari donne au corps un statut théorique et métapsychologique: ce ne sera donc ni le sein ou le non-sein, ni la frustration ou la gratification, ni la mère et ses fonctions qui seront à la source de l'activité de représentation mais plutôt la sensation brute elle-même telle qu'inscrite dans le corps du bébé ou de l'adulte. Il insiste sur les aspects physiologiques, que ce soit la faim, la chaleur, le froid ou le sommeil, qui agissent comme déclencheurs de l'expérience émotionnelle; la réponse subséquente, par le biais de la rêverie maternelle, arrive en second. La vie psychique devra affronter continuellement ce corps concret, sorte d'objet de névrose actuelle toujours active dans sa quête et sa transformation de l'expérience, même si le corps doit s'éclipser la plupart du temps pour laisser place à l'activité de représentation mentale.

\section{L'exemple de Virginie}

Quinodoz (2002) propose de façon très sensible d'utiliser un langage qui touche, un langage incarné faisant appel au registre sensoriel qui ouvre la possibilité de réveiller des fantasmes corporels et permette ainsi à l'analysant d'y trouver un sens émotionnel. «Si un analysant a besoin de récupérer des expériences affectives précoces, c'est par le biais de l'expérience corporelle qu'il pourra retrouver affects et fantasmes corporels (p. 56)». «Le langage qui touche passe par la mémoire de l'analyste», ajoute-t-elle, laquelle intervient comme un gardien dynamique de ce que le patient a déposé en nous. L'analyste accueillera ces sensations et pourra ensuite, après les avoir éprouvées dans son corps, les restituer au patient en essayant de leur donner une signification émotionnelle. L'identification projective peut alors être utilisée comme un moyen pour expulser non pas les affects mais leurs manifestations sensorielles qui sont celles dont il est le plus facile de devenir conscient.

Un exemple clinique tiré d'une analyse d'adulte servira ici de trame aux réflexions théoriques subséquentes.

Virginie avait un peu plus de 18 ans quand nous la rencontrons pour la première fois. Le travail analytique avec cette attachante jeune femme aura duré une dizaine d'années. Elle cultive volontairement, à ses yeux et à ceux des autres, une impression juvénile et une allure androgyne; elle camoufle sous des vêtements amples et peu féminins des seins menus et garde ses cheveux courts. Elle a des problèmes de poids, frôlant l'anorexie ou tombant parfois dans des excès boulimiques. Elle oscille quant à son orientation sexuelle et cherche aussi sa voie dans ses études: aussi brillante qu'elle ait toujours été au plan académique, elle a changé d'orientation à maintes reprises sans arriver à compléter les programmes choisis. Plus tard, c'est la «phobie de la puanteur» qui sera au centre du travail analytique.

$\grave{A}$ vingt ans, ses premières relations sexuelles avec son copain la laisse avec un mélange de «béatitude» et de "peur de se perdre ou de perdre son identité ». Elle 
craint de ne pas pouvoir être et vivre près d'un homme de façon soutenue, en raison de ses gaz intestinaux qui "empoisonneraient l'autre» ou que, par un contact physique rapproché, ne se révèle "son enveloppe de puanteur». La honte l'habite et restreint ses activités sociales déjà peu nombreuses; le contact avec les autres crée en elle le sentiment d'être vidée et accentue ses sentiments d'étrangeté, de différence et d'isolement. Elle ne comprend pas qu'un tel «chaos» puisse exister à l'intérieur d'elle, se disant qu'elle n'a pourtant pas connu de traumatisme important dans sa vie.

Sa puberté a mis du temps à arriver après deux ans de douleurs récurrentes au bas-ventre. À 14 ans, peu avant ses premières règles, un "rêve érotique et incestueux» lui a fait craindre d'être enceinte de son père ou de son frère et a marqué le début de son besoin de contrôler son poids, de peur de grossir. Les émois suscités par les baisers échangés avec un garçon n'ont alors fait qu'accrôtre sa vigilance par rapport à son corps et accentuer ses sentiments dépressifs.

Cette mise en contexte me permettra maintenant de me concentrer sur quelques moments de la dernière phase de son analyse au cours de laquelle je constaterai la fluctuation des niveaux de fonctionnement, passant de préoccupations névrotiques à d'autres, plus primitives. Ainsi, il s'est dégagé de «l'enveloppe de puanteur » des ramifications avec ses conceptions de l'enfantement et un sens confus de son identité personnelle, y compris sexuelle, lui laissant l'impression que sa vie n'était que chaos.

\section{Séance :}

Elle arrive angoissée à sa séance. En effet, la veille, son projet de sculpture en cours de réalisation a bloqué. Un joint de marijuana n'a fait que décupler son angoisse. Je l'invite alors à me parler de celle-ci, peu importe la forme qu'elle prend -sensations physiques ou images. Elle dit ressentir une agitation intérieure importante, un poids qui l'écrase et contre lequel elle n'arrive plus à bouger et elle évoque une grande confusion, un chaos intérieur qui se reflète même dans sa chambre où plus rien ne doit bouger ou être rangé. Elle dit ne plus pouvoir ni penser ni rêver et se sentir seule sur le divan.

Je pense alors à un phénomène ressenti de nombreuses fois en sa présence alors que je me trouvais envahi par un besoin quasi irrépressible de bouger durant les séances en face à face, sensations contenues à grand peine et que je n'arrivais pas à m'expliquer. J'établis soudain intérieurement un lien entre mon agitation et sa confusion. J'étais aux prises avec une angoisse ressentie à un niveau infraverbal qui cherchait à sortir par la voie musculaire, évacuation de choses tues, cachées ou monstrueuses, et encore impossibles à mettre en mots.

D’autres images angoissantes surgissent. Après un long silence, elle raconte qu'encore aujourd'hui et depuis l'adolescence, elle craint de faire des gaz qu'elle ne pourrait contrôler et de sentir mauvais. Cela la distrait tout le temps. À sa première année de cégep, à 16 ans, elle était envahie par ces peurs et elle guettait 
les réactions de dégoût sur les visages. Elle ne savait plus si les odeurs venaient "du dedans ou du dehors», la laissant confuse. Lui viennent encore aujourd'hui des images de porosité de son corps d'où les gaz s'échappent à son insu. Son anorexie, elle n'ingérait pas de nourriture, visait à la purifier jusqu'au point de ne plus avoir de gaz. Ceux-ci lui servaient à sentir mauvais et à repousser ceux par qui elle se sentait attirée. En contrepartie, elle avait trouvé un très bon parfum, devenu "sa marque de commerce», que tout le monde appréciait.

Elle ajoute que sa mère est très sensible aux odeurs; elle évoque le jeu de faire semblant de "manger un bébé» tellement il sent bon. Elle a des souvenirs d'intrusion et de forçage alimentaire commis par son père et par l'éducatrice de la garderie. L'idée lui vient alors que le monstre dessiné la veille est peut-être endedans d'elle, quelque chose de mauvais. Des souvenirs d'enfance s'ajoutent en rapport au corps et aux fonctions d'élimination. Sa mère aime raconter, sur un ton amusé, que Virginie, alors qu'elle était bébé, avait fait un caca débordant sur le canapé qui en a gardé une trace encore visible aujourd'hui. Virginie demeure honteuse devant ces incidents de souillure. Elle a le sentiment que, depuis fort longtemps, elle ne devait pas produire de caca mais, tout au contraire, que du bon.

Je me contente, pour ma part, de souligner la confusion entre intérieur et extérieur, la porosité appréhendée du corps et l'angoisse que suscite cette vision des frontières perçues comme instables et mobiles de la sorte, tout en songeant aux aspects primitifs de la personnalité. Le besoin de m'agiter a dès lors et depuis ce moment cessé en séance avec elle.

D'autres aspects de sa personnalité ont été abordés et de nombreuses références transgénérationnelles ont constitué des sillons maintes fois retracés et parcourus mais ils resteront dans l'ombre à ce moment-ci. Au cours des séances qui suivront, Virginie déploiera d'intenses efforts de créativité pour représenter sa personnalité en quête d'unification. Elle est souvent revenue à cette idée de frontières floues et mouvantes du corps lorsqu'il a été question des dangers de blessure lors de masturbations «avec la main sale» pratiquées depuis l'enfance, ou lors de relations sexuelles où elle craignait de se perdre. Elle se disait fatiguée d' «un corps qui n'est pas stable», fatiguée de craindre de perdre des morceaux comme dans ses rêves où des chats étaient mutilés ou perdaient leurs chatons.

\section{Séance suivante :}

Les gaz feront irruption dans la relation transférentielle. Suite à mon interprétation, elle arrive en retard le lendemain, du fait de ses craintes par rapport aux gaz, dit-elle. Elle "m'avoue», avec gêne, avoir eu, hier, une crainte d'avoir fait un gaz en séance et qu'elle a eu bien envie de me demander si ça sentait quelque chose pour se rassurer. Ce qui la trouble le plus, c'est qu'elle ne peut plus se fier à ses perceptions et qu'elle a besoin de la parole d'un autre pour désamorcer son angoisse dans ces moments-là.

La veille, au moment de l'inviter à entrer, j'avais cru sentir une odeur d'urine dans le corridor du bureau. J'étais donc bien en phase avec elle! J'interviens en 
associant alors sur la gêne de perdre sa contenance et que des gaz puissent s'échapper involontairement ou par inadvertance. Plusieurs questions me viennent à l'esprit qui prendront une forme de proposition libre en séance conduisant à un dialogue analytique tenu très près des sensations corporelles: Comment se représente-t-elle son intérieur? Qu'aurais-je pu penser d'elle si un gaz était sorti? L'aurais-je repoussée ou laissée tomber? Comment peut se sentir un bébé qui fait des gaz? Peut-il s'accrocher, aimer l'odeur de sa couche salie? Dans quel espace interne se font les bébés?

Elle répond par le biais d'une sensation rapportée d'un rêve de la nuit précédente, sensation fréquente par ailleurs chez elle, d'une "immense solitude». Son rêve de grossesse à l'adolescence ne l'a pas inquiétée par son aspect incestueux, dit-elle, mais plutôt du fait d'avoir une bosse au bas du ventre. Pour elle, les règles augmentaient les gaz, son ventre se gonflant avant les règles. Je dis alors que les frontières entre les intestins et l'utérus semblent s'embrouiller au point de communiquer ensemble. Elle n'avait jamais réfléchi à tout cela en considérant les choses sous cet angle. Même si, adolescente, des livres étaient disponibles pour savoir «comment se font les bébés», elle n'avait jamais rien trouvé à lire sur sa "phobie des odeurs».

Mes remarques, volontairement «hors transfert» et centrées sur son corps, faisaient appel à mon contre-transfert très ancré dans un effort identificatoire avec elle sur ce que pouvait être une confusion des espaces intérieurs et des fonctions corporelles, tout en suivant de près ses propres explorations. Quelques jours plus tard, son travail de bénévole avec des suicidaires la rapprochera de cette partie d'elle qui a voulu mourir à l'adolescence.

\section{Séance :}

Une image lui vient où elle se voit couchée sur un lit en position fœtale, alors qu'elle doit sauver une fille suicidaire. Un silence s'installe en séance : elle craint d'avoir fait un gaz. C'est angoissant, dit-elle, de ne plus pouvoir faire confiance à ses sens. Elle se sent «poreuse, comme dissoute ou dissoluble». Je commente simplement en verbalisant une image que j'avais alors, soit celle «d'un fœtus qui se sent perdu dans un espace infini». Elle acquiesce et ajoute, «les gaz s'expandent et je perds alors mon intégrité ; ce n'est pas seulement physique, c'est psychique aussi ».

Malgré l'angoisse suscitée, ces états la font rêver et créer, dit-elle. Ainsi, elle a imaginé une installation artistique où elle représenterait des intestins à vue, tout en blanc, purs, dans un corps étendu sur un lit. Une mèche de tissu blanc irait de l'intestin vers une fenêtre par où sortirait le tissu, pour représenter le lien entre l'intérieur et l'extérieur, et ainsi symboliser les territoires réunis.

Les angoisses primitives ont souvent pour effet de délier les perceptions et les fonctions corporelles correspondantes, cette déliaison devenant source de confusion. Le système projectif manque de force et s'arrête au corps, faute d'avoir pu être traité par un objet maternel suffisamment adéquat. C'est à ce moment que 
les tentatives de projection trouveront un terreau fertile dans ou sur le corps qui devient par défaut un objet chargé d'un fort investissement narcissique.

Le cas de Virginie illustre le propos de Freud (1923) dans «Le moi et le ça» alors qu'il développe la notion de Moi corporel: «Le corps propre, et avant tout sa surface, est un lieu dont peuvent provenir simultanément des perceptions internes et externes.» Cette courte phrase contient deux idées importantes concernant le corps : celle de sa surface comme image et celle de barrière ou frontière opérant la distinction entre intérieur et extérieur. Nous savons que cette double source d'informations peut engendrer de la confusion pour le sujet. En partant de l'image d'un bébé dont la peau fragile est «criblée» d'ouvertures, - yeux, nez, oreilles, bouche, pores de la peau, zone uro-génitale — dont il n'a pas le plein contrôle sur l'ouverture ou la fermeture, nous pouvons spéculer que le bébé ait l'impression que le contenu de son corps aux parties encore mal différenciées risque de se répandre involontairement au dehors ou que ces mêmes ouvertures pourraient à l'inverse laisser entrer des sensations inattendues et que celles-ci persistent longtemps et même à l'âge adulte.

Un long travail préparatoire avec Virginie nous a permis d'aller au plus près de ses angoisses primitives dont les plus alarmantes concernaient sa crainte de se transformer en gaz, de se diffuser par porosité, angoisses qui se répercutaient sur son sentiment d'exister, d'être, sur son identité sexuelle et professionnelle et sur ses doutes quant à sa capacité à créer. Notre travail sur les parties de soi éparpillées et le flou de ses propres frontières a été le plus aidant pour elle, dira-t-elle à la fin. Meltzer (1971), dans «Le processus psychanalytique», insiste sur l'importance de réaliser un consensus des sens et le rassemblement de perceptions fractionnées. Dans ce cas, Virginie n'arrivait pas à donner un sens à ses sensations physiques et c'est ce qui l'a poussée à effectuer un intense travail de mise en images puis en mots de celles-ci.

Cette pulsation pulsionnelle exercée par une ou des sensations traversant le corps peut être investie et devenir une source d'expression et de travail psychique. Virginie l'illustre par ses tentatives de créer des représentations visuelles sur lesquelles elle s'appuie pour trouver un sens à ses gaz. Comme l'image du rêve, elle nécessitera un second travail d'élaboration avant de l'amener à un niveau d'abstraction et de représentation suffisant pour lui procurer un niveau de confort dans un espace interne sécuritaire. Si Freud (1923) soulignait l'importance des restes mnésiques optiques en tant que voie privilégiée dans le processus du passage inconscient-conscient de la pensée, Gaddini (1982) précise davantage les étapes inhérentes à ce processus, alors que les représentations dans le corps précèdent celles, plus avancées, qui passent par la voie de l'image.

Nous sommes familiers avec la perte momentanée du lien entre le corps et ses fonctions telle qu'elle s'exprime dans la dépersonnalisation. Winnicott (1971) a pour sa part introduit l'idée d'une «personnification», soit l'habitation de son corps relié à la psyché, mais aussi à ses besoins instinctuels. Qu'il s'agisse de Virginie ou de Denis, la mise en mots des angoisses primitives a constitué une 
route pour reconstituer et rapatrier ces liens entre le corps et la psyché, vers une personnification plus cohérente et solide.

J'abonde dans le sens de Lombardi (2002) quand il affirme que le corps peut être pris comme point de départ du fonctionnement mental, pour passer de la sensation à la pensée tel qu'esquissé ici dans le travail avec Virginie ou Denis. Le travail interprétatif se déplace alors sur le transfert fait sur les objets internes de l'analysant, alors représentés par des parties ou des fonctions corporelles, plutôt que sur les aspects plus généraux d'un transfert classique réactualisant des scènes du passé ou sur l'analyste lui-même, éléments du transfert qui, sans être exclus, seront abordés à d'autres moments choisis.

Ferrari (2004) prône une traduction par l'analyste des communications inconscientes de l'analysant en un langage accessible à la conscience, ce qui suppose que l'analyste dispose d'une clarté intérieure à propos des enjeux en cause. La difficulté consiste à trouver un langage qui ne soit ni réducteur ni infantilisant, ni rapportable à une position ou un savoir dénotant la toute-puissance. Pour ce faire, l'analyste a besoin de s'appuyer sur sa capacité de «rêverie maternelle» suffisamment intériorisée au décours de ses expériences. À cet égard, le travail psychique réalisé lors des observations de bébés et dans les traitements parentsbébé m'ont grandement aidé à pratiquer cette disponibilité intérieure bien particulière. «L'analyste devient une sage-femme permettant de donner naissance aux pensées qui cherchent à naître », comme le note Lombardi (2008).

Il y a des implications techniques à donner une telle place au corps durant les séances. L'analyste accepte alors que l'individu vive plus pleinement cette relation à lui-même suivant cet axe corps-esprit et par la suite, ses interventions seront davantage dirigées de façon à favoriser l'émergence de prises de conscience intégrant des aspects corporels. Cette façon de faire initiale permet d'aborder autrement les aspects somatiques en séance, qu'il s'agisse de problèmes psychosomatiques comme tels ou de problématiques anxieuses pour lesquelles les répercussions sur le corps sont souvent omniprésentes et inévitables, voire incontournables en séance. Si le corps peut devenir le berceau des expériences émotionnelles, il risque aussi d'en être le tombeau lorsque le corps implose sous la pression de sensations vécues comme des corps étrangers qui font déborder les capacités de contenance psychique du sujet.

\section{Conclusion}

Que représente la césure de la naissance et qu'en résulte-t-il ? C'est le début d'un long travail pour le bébé naissant et grandissant que deux tâches attendent: l'une pour devenir conscient de son propre corps et des phénomènes sensoriels qui l'habitent et qui, parfois, le désorganisent et l'autre qui consistera à distinguer ce qui appartient au corps de sa mère pour arriver à voir celle-ci comme un objet extérieur et séparé de son corps à lui.

Au cours des dernières décennies, les théories psychanalytiques ont fait une très large place à la compréhension, certes essentielle et vitale, des relations précoces 
parents-enfants et au développement des relations d'objet. Parallèlement, ont paru des études portant sur le développement des bébés pris isolément sans tenir compte de ces mêmes relations et qui ont révélé des capacités précoces diverses et étonnantes sur les plans corporel, affectif, cognitif, relationnel et sensoriel. Ces deux mondes s'opposent encore mais des voix tentent de rapporter ces résultats de recherche psychanalytique et développementale de façon à pouvoir travailler autrement et surmonter ainsi certaines des embûches rencontrées en psychanalyse, peu importe l'âge des patients.

D'autre part, des positions souvent inconciliables s'affrontent et sont entretenues à l'intérieur même du mouvement psychanalytique en ce qui concerne la dualité entre corps et esprit, incluant celle ayant trait à la prégnance de la réalité psychique par opposition au monde extérieur et aussi celle de la transformation d'éléments non pensés en éléments nourrissants pour nos personnalités. Une cure psychanalytique devrait permettre de confronter ces dualités apparentes et leur offrir un travail d'élaboration et de liaison approprié. Une approche laissant une place au corps peut permettre à ces paradoxes de se déployer au cours d'un traitement sans avoir l'impression d'en négliger ou d'en surexposer un au détriment d'un autre. Il peut ainsi se développer un va-et-vient entre les éléments d'ordre sensoriel, ceux relevant des émotions et ceux témoignant plus directement de l'activité de la pensée. La capacité de «rêverie maternelle» suivant Bion servira alors d'articulation véritable entre le monde relationnel essentiel à la vie et les matériaux perceptuels, avec la prise en compte par l'analyste des données neurophysiologiques propre au bébé, à l'enfant ou à l'adulte. De là, le développement d'une expérience émotionnelle authentique pourra agir comme intermédiaire et fil conducteur entre ce qui, des traces de la sensation physique, sera investi et deviendra une pensée mentalisée.

L'origine de toute individualité, sa source, est, de façon incontournable, multiple avec à sa base un homme et une femme qui ont mis en commun quelque chose d'eux-mêmes pour créer un «autre» humain. C'est sur ce socle que s'édifiera un sujet qui agit et qui pense, alimenté par la force vitale et par le dynamisme des liens se tissant entre un père, une mère et un bébé. Mais pour advenir au monde et à lui-même, ce bébé-source, ce bébé-jaillissement, avec et malgré son indifférenciation, son inorganisation, aura besoin aussi, pour y arriver, de prendre possession de cette «résidence», celle d'un corps habité de sa psyché.

alain lebel

951, ave laporte montréal $\mathrm{h} 4 \mathrm{c} 2 \mathrm{p} 6$ alebela@videotron.ca 
Filigrane, printemps 2009

\section{Bibliographie}

Athanassiou, C., 1982, La constitution et l'évolution des premières identifications, Revue française de psychanalyse, XLVI, 6, 1187-1209.

Athanassiou, C., 1997, L'identification adhésive: la contribution de ce concept à la compréhension des états eczémateux, Journal de psychanalyse de l'enfant, 215-244.

Anzieu, D., 1985, Les fonctions du Moi-peau, in Le Moi-peau, Dunod, deuxième édition (1995).

Balestrière, L., 2008, Freud et la question des origines, Bruxelles, de Boeck, troisième édition.

Bégoin, J., 1989, La violence du désespoir ou le contresens d'une pulsion de mort en psychanalyse, Revue française de psychanalyse, LIII, 2, 619-640.

Bick, E., 1967, L'expérience de la peau dans les relations d'objet précoces, in Les écrits de Martha Harris et d'Esther Bick, Éditions du Hublot (1998).

Bion, W. R., 1957, Differentiation of the psychotic from the non-psychotic personalities, International Journal of Psychoanalaysis, 38, 266-275.

Bion, W. R., 1962, Aux sources de l'expérience, Paris, PUF.

Bizzozero, V., 2007, En deça du pulsionnel : réflexions autour des sources physiologiques et sensorielles de l'appareil psychique, Éditions Médecine \& Hygiène, Genève.

Ciccone, A., L'hôpital, M, 2001, Naissance à la vie psychique. Modalités du lien précoce à l'objet au regard de la psychanalyse, Paris, Dunod, deuxième édition.

Ferrari, A. B., 2004, From the Eclipse of the Body to the Dawn of Thought, London, Free Association Books.

Freud, S., 1895, Esquisse d'une psychologie scientifique, in Naissance de la psychanalyse, Paris, PUF (1996).

Freud, S., 1915, Pulsions et destin des pulsions, in Métapsychologie, Gallimard (1968), p. 11-43.

Freud, S., 1917, Deuil et mélancolie, in Métapsychologie, Gallimard (1968), 144-171.

Freud, S., 1923, Le Moi et le Ça, in Essais de psychanalyse, Paris, Payot (1981).

Freud, S., 1938, Abrégé de psychanalyse, Paris, PUF.

Gaddini, E., 1982, Early defensive fantasies and the psychoanalytical process, International Journal of Psychoanalysis, 63, 379-388.

Haag, G., 2004, Sexualité orale et Moi corporel, Topique, 87, 23-45.

Haag, G., 1997, Contribution à la compréhension des identifications en jeu dans le moi corporel, Journal de la psychanalyse de l'enfant, 20, Le corps, Éditions Bayard.

Haag, G., 1991, Nature de quelques identifications dans l'image du corps, Journal de psychanalyse de l'enfant, 10, 73-92.

Haag, G., 1984, Réflexions sur les premiers niveaux d'identification, in Travail de la métaphore, Denoël.

Haag, M., 2002, À propos et à partir de la personne d'Esther Bick, Paris, autoédition.

Klein, S., 1980, Autistic phenomena in neurotic patients, International Journal of Psychoanalysis, 61, 395-402.

Lebel, A., 2008, Accroître la fonction contenante des psychothérapeutes par l'expérience de l'observation des nourrissons selon la méthode d'Esther Bick, Filigrane, 17, 1, 105-120.

Lebel, A., 2000, Mouvements de vie, PRISME, 31, 34-51.

Lombardi, R., 2008, The body in the analytic session: Focusing on the body-mind link, International Journal of Psychoanalysis, 89, 89-110.

Lombardi, R., 2002, Primitive mental states and the body : a personal view of A.B. Ferrari's concrete original object, International Journal of Psychoanalysis, 83, 363-381.

Meltzer, D., 1971, Le processus psychanalytique, Paris, Payot. 
Meltzer, D., 1975, Explorations dans le monde de l'autisme (traduction française, 1980), chapitre IX, Payot.

Meltzer, D., 1984, Les concepts d'identification projective (Klein) et de contenant-contenu (Bion) en relation avec la situation analytique, Revue française de psychanalyse, 28, 2, 541-550.

Mitrani, J., 2008, The comments of Dr Vartzopoulos (Cf Mitrani, 2007), International Journal of Psychoanalysis, 89, 660-662.

Mitrani, J., 2007 Bodily centred protections in adolescence : An extension of the work of Frances Tustin, International Journal of Psychoanalysis, 88, 1153-1169.

Ogden, T., 1994, The analytic third: working with intersubjective clinical facts, International Journal of Psychoanalysis, 75, 3-18.

Ogden, T., 2001, Re-minding the body, American Journal of Psychotherapy, 55, 1, 92-104.

Quinodoz, D., 2002, Des mots qui touchent, PUF.

Winnicott, D. W., 1945, Le développement affectif primaire, Petite Bibliothèque Payot (1969).

Winnicott, D. W., 1949, L'esprit et ses rapports avec le psyché-soma, Petite Bibliothèque Payot (1969).

Winnicott, D. W., 1952, Psychose et soins maternels, Petite Bibliothèque Payot (1969).

Winnicott, D. W., 1971, Le corps et le self, Nouvelle Revue de psychanalyse, 3, 37-48. 